\title{
NO Forms an Adduct with Serum Albumin that Has Endothelium-derived Relaxing Factor-like Properties
}

\author{
John F. Keaney, Jr., Daniel I. Simon, Jonathan S. Stamler, Omar Jaraki, \\ Jonathan Scharfstein, Joseph A. Vita, and Joseph Loscalzo \\ Department of Medicine, Brigham and Women's Hospital, Brockton/West Roxbury Veterans \\ Administration Medical Center, Harvard Medical School, Boston, Massachusetts 02132
}

\section{Abstract}

Recent evidence suggests that sulf hydryl species can react with oxides of nitrogen under physiologic conditions and thereby stabilize endothelium-derived relaxing factor (EDRF) activity, but the presence of a specific in vivo thiol carrier for nitric oxide (NO) remains controversial. The single free sulfhydryl of serum albumin is the most abundant thiol species in plasma $(\approx 0.5 \mathrm{mM})$ and is particularly reactive towards NO. To examine the potential role of serum albumin in endogenous nitric oxide metabolism, we synthesized $S$-nitroso-BSA (S-NOBSA ), a model $S$-nitroso-protein, and examined its effects on platelet function and coronary and systemic vascular tone in $\mathbf{1 6}$ mongrel dogs. Intravenous bolus S-NO-BSA markedly reduced mean arterial pressure in a dose-dependent manner and proved seven and a half-fold less potent than intravenous nitroglycerin and 10 -fold less potent than intravenous $S$-nitroso-cysteine ( half-maximal response of $75 \mathrm{nmol} / \mathrm{kg}$ compared to 10 and 7.5 nmol $/ \mathrm{kg}$, respectively; $P<0.05$ ); when given by intravenous infusion (half-maximal response $=10 \mathrm{nmol} / \mathrm{kg}$ per min), however, $S$-NO-BSA and nitroglycerin were equipotent. Intravenous bolus $S$-NO-BSA had a greater duration of action than either nitroglycerin or $S$-nitroso-cysteine and produced marked prolongation of the template bleeding time associated with dose-dependent inhibition of ex vivo platelet aggregation (halfmaximal response $\sim 70 \mathrm{nmol} / \mathrm{kg}$ ). Intracoronary $S$-NO-BSA increased coronary blood flow (mean \pm SEM) less effectively than nitroprusside, acetylcholine, or $S$-nitroso-cysteine (165\% $\pm 24 \%$ vs. $315 \% \pm 82 \%, 483 \% \pm 55 \%$, or $475 \% \pm 66 \%$, respectively; $P<0.05$ ) although with much longer duration of action. On a molar basis, $S$-nitroso-cysteine proved more effective than $S$-nitroso-BSA, nitroprusside, or acetylcholine as an epicardial coronary vasodilator. Thus, serum albumin reacts with oxides of nitrogen to form a stable $S$-nitrosothiol with properties reminiscent of authentic EDRF supporting the view that protein associated thiol may participate in the action and metabolism of EDRF. (J. Clin. Invest. 1993. 91:1582-1589.) Key words: nitric oxide $\bullet$ proteins $\bullet$ thiols $\bullet$ endothelium-derived relaxing factor $\bullet$ platelets

Part of this work has been presented in abstract form at the 64th Scientific Sessions of the American Heart Association, Anaheim, CA, 11-14 November 1991.

Address correspondence to Joseph Loscalzo, M.D., Ph.D., Department of Medicine, Cardiovascular Division, Brigham and Women's Hospital, 75 Francis St., Boston, MA 02115.

Received for publication 3 October 1992 and in revised form 12 October 1992.

The Journal of Clinical Investigation, Inc.

Volume 91, April 1993, 1582-1589

\section{Introduction}

The vascular endothelium is important in a number of homeostatic functions including the regulation of blood flow, maintenance of vascular tone, and the balance between thrombosis and blood fluidity. In 1980, Furchgott and Zawadzki (1) described a potent vasodilator produced by the endothelium later termed endothelium-derived relaxing factor (EDRF). ${ }^{1}$ This endothelial product activates guanylyl cyclase in vascular smooth muscle cells and platelets, leading both to vasorelaxation and platelet inhibition $(2,3)$. The chemical nature of EDRF has been examined in detail with the preponderance of evidence now suggesting that EDRF is chemically related to the nitric oxide radical (NO) (4).

Nitric oxide is a highly reactive molecule that readily combines with a number of biochemical species, including heme iron, nonheme iron (5), oxygen (6), and superoxide anion (7), forming a variety of derivative oxides of nitrogen (6). These oxides of nitrogen are themselves reactive and form adducts with readily available intra- and extracellular species, including thiols (8-10), (primary) amines (11), and unsaturated fatty acyl groups (12). The reactive nature of NO limits the reproducibility with which free NO can activate guanylyl cyclase and may, in part, explain the inconsistent observations equating EDRF and NO (13-18).

Biologic thiols react readily with oxides of nitrogen forming $S$-nitrosothiols (8-10) that possess chemical half lives substantially longer than that of NO, e.g., $S$-nitroso-L-cysteine has a half-life under physiologic conditions $\left(37^{\circ} \mathrm{C}, \mathrm{pH} 7.4\right)$ of $15-30$ s (19), while that of NO is only $0.1-1$ s (16). $S$-nitrosothiols have been shown to prolong the physiologic half-life of NO (17), inhibit platelets through a cGMP-dependent mechanism (10), and possess EDRF-like vasorelaxant properties (20).

Despite these well-characterized interactions between biologic thiols and derivative oxides of nitrogen, the presence of a specific thiol carrier for nitric oxide in vivo remains controversial. Moreover, the potential contributions of protein-bound thiol in the action and metabolism of nitric oxide have been largely overlooked. Protein thiol represents the most abundant form of thiol in plasma and is particularly prevalent in the cellular cytosol (21). In this regard, we have recently demonstrated that oxides of nitrogen can react with protein sulfhydryl groups under physiologic conditions producing stable, biologically active $S$-nitroso-proteins that possess potent vasorelaxant and platelet inhibitory properties in vitro (22). We report here the in vivo properties of $S$-nitroso-BSA, a model $S$-nitroso pro-

1. Abbreviations used in this paper: CPD, citrate-phosphate-dextrose anticoagulant solution; $\mathrm{EC}_{50}$, half-maximal response; $\mathrm{EDRF}$, endothelium-derived relaxing factor; NO, nitric oxide. 
tein, in the coronary and systemic vascular beds and its effects on bleeding time and ex vivo platelet aggregation.

\section{Methods}

Materials. Sodium nitrite was purchased from Fisher Scientific (Fairlawn, NJ). Sulfanilamide and $\mathrm{N}$-(1-naphthyl)ethylenediamine dihydrochloride were obtained from Aldrich Chemical Co. (Milwaukee, WI). Isoflurane was obtained from Anaquest Co. (Madison, WI). Collagen was purchased from Worthington Biochemical Corp. (Freehold, NJ). Bleeding time templates were purchased from Organon Teknika Corp. (Durham, NC). BSA, L-cysteine, timolol maleate, atropine sulfate, hexamethonium bromide, adenosine diphosphate, and all other materials were obtained from Sigma Chemical Co. (St. Louis, MO).

Tris-buffered saline consisted of $10 \mathrm{mM}$ tris[hydroxymeth-

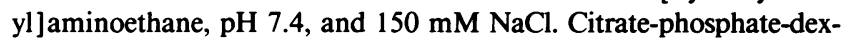
trose anticoagulant solution (CPD) contained $3 \mathrm{~g}$ /liter citric acid, 26.3 $\mathrm{g} /$ liter sodium citrate, $2.22 \mathrm{~g} /$ liter $\mathrm{NaH}_{2} \mathrm{PO}_{4} \cdot \mathrm{H}_{2} \mathrm{O}$, and $25.6 \mathrm{~g} /$ liter dextrose, at $\mathrm{pH} 7.35$.

Synthesis of $S$-nitroso-species. S-nitroso-BSA was synthesized as previously described (22). Briefly, BSA was exposed to NO generated from equimolar $\mathrm{NaNO}_{2}$ in $0.5 \mathrm{~N} \mathrm{HCl}$ for a period of $30 \mathrm{~min}$ at room temperature and neutralized with an equal volume of TBS and $0.5 \mathrm{~N}$ $\mathrm{NaOH}$. The formation of $S$-nitroso-BSA was confirmed by both chemical and spectrophotometric methods. The content of $S$-nitrosothiol was determined by the method of Saville (23), in which NO elaborated from $S$-nitrosothiol species with $\mathrm{Hg}^{2+}$ is assayed by diazotization with sulfanilamide and coupling with the chromophore $N$-( 1-naphthyl-)ethylenediamine. Comparison of the sample absorbance at $550 \mathrm{~nm}$ with a known standard allows quantification of displaceable NO, which is directly derived from $S$-nitrosothiol. The presence of an $S$-nitrosothiol bond was confirmed with $\left[{ }^{15} \mathrm{~N}\right]$ nuclear magnetic resonance spectroscopy by a distinctive chemical shift of the observed peak (751 ppm relative to nitrite [24]). Typical $S$-nitrosothiol absorption maxima at $320-360$ and $550 \mathrm{~nm}$ provided additional confirmatory evidence for the presence of an $S$-nitrosothiol bond. Synthesis of $S$-nitrosoBSA by this method produced a stoichiometry of $S$-NO/BSA of $0.85 \pm 0.04$ (SEM).

$S$-nitroso-cysteine was synthesized by exposure of equimolar L-cysteine and $\mathrm{NaNO}_{2}$ in $0.5 \mathrm{~N} \mathrm{HCl}$. The presence of an $S$-nitrosothiol was confirmed by the method of Saville (23). Synthesis of $S$-nitroso-cysteine by this method results in a product that is $99.9 \%$ pure (25) and stable in an acidic environment $(0.5 \mathrm{~N} \mathrm{HCl})$. Immediately before in vivo administration, each dose of $S$-nitroso-cysteine was neutralized with $10 \mathrm{vol}$ of Tris-buffered saline, $\mathrm{pH} 7.0$.

Determination of plasma S-nitrosothiol content. Plasma (4 ml) was centrifuged (with $10 \%$ [ vol/vol] CPD) at $800 \mathrm{~g}$ for $10 \mathrm{~min}$ and stored at $-70^{\circ} \mathrm{C}$. The determination of plasma protein and low molecular weight $S$-nitrosothiol content was performed as previously described (26). Briefly, plasma $(100 \mu \mathrm{l})$ is injected into a specially designed chemiluminescence detector (Thermedics Inc., Woburn, MA), carried into a glass coil with a purge stream of helium, and is irradiated with ultraviolet light ( $300-400 \mathrm{~nm}$ ). NO, cleaved homolytically by photolysis, is separated rapidly from the solvent through a series of cold traps and is carried by the helium into the reaction chamber of the chemiluminescence detector. The passage of intact plasma through the chemiluminescence detector without ultraviolet light affords the measurement of free NO while total NO content (free NO $+S$-nitrosothiol) is determined by chemiluminescence after ultraviolet photolysis. Chemiluminescent analysis of the supernatant from plasma protein precipitates provides quantification of low molecular weight $S$-nitrosothiol. The plasma protein $S$-nitrosothiol content is then deduced from the difference between total plasma NO content and the plasma content of low molecular weight $S$-nitrosothiol and free NO.

Animal preparation. 16 mongrel dogs of either sex weighing 20-26 $\mathrm{kg}$ were anesthetized with thiamylal sodium, $25-30 \mathrm{mg} / \mathrm{kg}$ i.v., intubated with a cuffed endotracheal tube, and ventilated with $50 \%$ oxygen ( 22 breaths $/ \mathrm{min}, 10-12 \mathrm{ml} / \mathrm{kg}$ stroke volume) and $1 \%$ isoflurane via a respirator (Drager AV; North American Drager, Telford, PA). Body temperature was monitored using a rectal probe and maintained with a homeothermic blanket (Harvard Apparatus, Edinbridge, KY). Oxygen tension $\left(\mathrm{PO}_{2}\right)$, carbon dioxide tension $\left(\mathrm{PCO}_{2}\right)$, and $\mathrm{pH}$ were monitored using a blood gas analyzer (model ABC 30; Radiometer America Co., Cleveland, $\mathrm{OH}$ ) and adjusted by manipulation of stroke volume

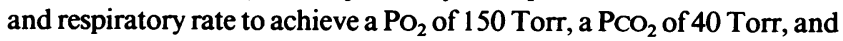
a pH of 7.40. The left and right femoral arteries were cannulated percutaneously with 9 French and 6 French sidearm sheaths, respectively (Cordis Corp., Miami, FL), and 6 French sheaths were placed in the right and left femoral veins. A 5 French high fidelity pressure catheter (model SPC-350; Millar Instrument Co., Houston, TX) was placed into the descending aorta via the 6 French sidearm sheath for continuous monitoring of arterial pressure. Cutaneous electrocardiographic leads were placed on all four extremities and an electrocardiogram recorded continuously throughout the protocol. The bladder was catheterized with a 6F urethral catheter (C. R. Bard Co., Covington, GA), and urine was drained into a graduated collection bag.

Intracoronary studies. In preparation for intracoronary drug infusions, the left circumflex coronary artery was cannulated with an $8 \mathrm{~F}$ Judkins-type guiding catheter (model FL2.5; Interventional Medical Co., Danvers, MA ). A 3F single-lumen, Doppler-tipped, velocity catheter (model DC-201; Millar Instruments) was advanced through the guiding catheter and the tip positioned $\sim 2 \mathrm{~cm}$ into the circumflex coronary artery for blood velocity measurement and selective drug delivery. A $5 \mathrm{~F}$ bipolar pacing catheter (C. R. Bard Co.) was positioned in the right ventricular apex and set in demand mode at $10 \mathrm{bpm}$ below the resting heart rate. Electrocardiogram, arterial pressure, and coronary blood velocity were continuously recorded on a physiograph (model 2600; Gould Electronics Co., Columbus, $\mathrm{OH}$ ). Catheter placement was documented by fluoroscopy before each intracoronary drug infusion.

After catheter placement, animals were allowed to stabilize for $\sim 20 \mathrm{~min}$. Acetylcholine, sodium nitroprusside, $S$-nitroso-cysteine, and $S$-nitroso-BSA were serially infused into the circumflex coronary artery at $0.8 \mathrm{ml} / \mathrm{min}$ for $3 \mathrm{~min}$. Control infusions included vehicle ( $5 \%$ dextrose in water for acetylcholine and nitroprusside), BSA, L-cysteine, and acidified and neutralized $\mathrm{NaNO}_{2}$. At the end of each 3-min infusion, arterial pressure, coronary blood velocity, and heart rate were recorded, and coronary angiography performed by the intracoronary injection of $7 \mathrm{ml}$ meglumine diatrizoate and sodium diatrizoate ( $\mathrm{Hy}-$ paque-76; Winthrop Pharmaceuticals Inc., New York). Coronary angiograms were recorded on 35-mm cine-angiographic film (Eastman Kodak Co., Rochester, NY) using a cine-angiographic system (Philips Medical Systems Inc., Raynham, MA).

Quantitative coronary angiography. Analysis of arterial dimensions was determined using an automated, previously validated quantitative angiography system (27-31). Technically suitable coronary angiograms with proximal, midvessel, and distal segments of the left circumflex coronary artery free of side branches were selected for quantitative analysis. For each drug infusion, two sites ( $\geq 7 \mathrm{~mm}$ in length) were subjected to analysis, the first located at the tip of the Doppler catheter and the second at a suitable distal site. The segment of interest was centered and the single-frame cine image digitized (20-40 microns/ pixel) with the use of a video camera (Cohu Inc., San Diego, CA) and a video interface (Recognition Concepts Inc., Incline Village, NV) connected to a computer (Micro VAX II; Digital Equipment Corp., Maynard, MA). Two-line profile averaging was used to minimize anatomical noise, and 16 video frames were summed to minimize video noise. Four cine frames at end-diastole were scanned and averaged using two anatomic features to ensure accurate alignment. Calibrated grids, filmed at isocenter, were used to scale data from pixels to millimeters, and fixed coordinates were used to reproduce regions of interest and assess serial responses. Coronary vasodilation is defined as the increase in vessel diameter (as a percent of the control value) in response to the infusion of a vasoactive agent. The analysis of coronary dimensions using this method reliably detects a $4 \%$ change in the arterial diameter of a 3-mm vessel (27). 
Analysis of changes in coronary blood flow. Coronary blood flow was estimated by the product of mean coronary blood velocity and coronary artery cross-sectional area at the Doppler catheter tip. Changes in coronary blood flow in response to drug infusions were determined from changes in mean coronary blood velocity corrected for alterations in cross-sectional area at the Doppler catheter tip. The limitations of the Doppler catheter preclude direct determination of coronary blood flow $(18,32)$.

Hemodynamic measurements. After completion of intracoronary infusions, the intracoronary catheters were removed and a bilateral vagotomy was performed through a midline neck incision. Reflex responses were blocked with $0.2 \mathrm{mg} / \mathrm{kg}$ i.v. atropine sulfate, $1 \mathrm{mg} / \mathrm{kg}$ i.v. timolol maleate, and $20 \mathrm{mg} / \mathrm{kg}$ i.v. hexamethonium bromide administered over $20 \mathrm{~min}$. Infusions of timolol maleate $(0.06 \mathrm{mg} / \mathrm{kg}$ per h $)$ and hexamethonium bromide $(10 \mathrm{mg} / \mathrm{kg}$ per $\mathrm{h})$ were continued throughout the remainder of the protocol. A stabilization period of $45 \mathrm{~min}$ separated the intracoronary infusions and the measurement of hemodynamic responses to $S$-nitroso-BSA. A 5 French high fidelity pressure catheter (model SPC-350; Millar Instrument Co.) was placed in the left ventricle from the femoral approach and connected serially to a differentiator and transducer (Gould Electronics Co.) for monitoring left ventricular pressure and its first derivative $(\mathrm{dP} / \mathrm{dt})$. A third 5 French pressure catheter (Millar) was placed in the pulmonary artery and a 7 French balloon-tipped catheter (Arrow International Inc., Reading, PA) was placed in the pulmonary capillary wedge position. Transducers were calibrated each morning and zero balance documented before each vasoactive drug administration. Nitroglycerin and $S$-nitroso-BSA were administered both as an intravenous bolus and a 5-min infusion. $S$-nitroso-cysteine was administered only as an intravenous bolus owing to its short half-life in aqueous solution and in plasma. Arterial pressure, heart rate, left ventricular pressure, left ventricular $\mathrm{dP} / \mathrm{dt}$, pulmonary artery pressure, and pulmonary capillary wedge pressure were recorded on a Gould physiograph, and a new control state established before any subsequent drug administration. The order of drug administration was chosen at random in order to control for confounding cumulative drug effects. Plasma samples for protein and low molecular weight $S$-nitrosothiol content were obtained before and after the administration of $S$-nitroso-cysteine.

Bleeding time and ex vivo platelet aggregation. In a subset of five animals, the bleeding time response to $S$-nitroso-BSA was examined. $S$-nitroso-BSA $(1,10,50$, and $150 \mathrm{nmol} / \mathrm{kg})$ was given as an intravenous 5 -min infusion followed in $15 \mathrm{~min}$ by a template bleeding time and determination of ex vivo platelet aggregation. Template bleeding times were performed on the ventral aspect of the tongue so as to minimize temperature fluctuation. Platelet aggregations were performed in platelet-rich plasma prepared by centrifuging whole blood (with $10 \%$ (vol/vol) CPD) at $150 \mathrm{~g}$ for $10 \mathrm{~min}$ at $25^{\circ} \mathrm{C}$. Platelet-poor plasma was prepared by centrifuging whole blood (with $10 \%$ [ $\mathrm{vol} / \mathrm{vol}$ ] CPD) at $800 \mathrm{~g}$ for $10 \mathrm{~min}$. Platelet counts were measured with a Coulter Counter (model ZM; Coulter Electronics, Hialeah, FL) and were adjusted to 150,000 platelets/ $\mu$ l by the addition of platelet-poor plasma. Platelet aggregations were performed with $400 \mu \mathrm{l}$ platelet-rich plasma at $37^{\circ} \mathrm{C}$ in an aggregometer (PAP-4 Biodata, Hatboro, PA), to which was added $10 \mu \mathrm{M}$ ADP or $0.24 \mathrm{mg} / \mathrm{ml}$ fetal calf skin collagen.

Statistical analysis. All data are presented as the mean \pm SEM unless otherwise indicated. The duration of action of an agent is defined as the time period during which the coronary blood flow exceeds $10 \%$ of the baseline value or, in the case of mean arterial pressure, the time period in which mean arterial pressure remained $\geq 10 \%$ below the baseline value. Dose-response curves for coronary blood flow, coronary vasodilation, and hemodynamic responses are compared on the basis of the concentration required to produce a half-maximal response $\left(\mathrm{EC}_{50}\right)$. The $\mathrm{EC}_{50}$ values presented were determined using the $\mathrm{E}_{\max }$ and $\mathrm{E}_{\min }$ values obtained at the maximum and minimum doses tested. Statistical comparison of dose-response curves for coronary vasodilation, coronary blood flow, and hemodynamic responses were performed by multivariate analysis of variance using a repeated measures design. Time-dependent responses of vasoactive agents, as well as bleeding

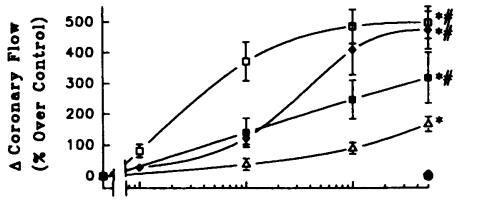

Figure 1. The coronary vascular response to $S$ nitroso-BSA. The effect of intracoronary $S$-nitroso-BSA $(\Delta)$, sodium nitroprusside $(\square)$, acetylcholine (ם), and $S$ -

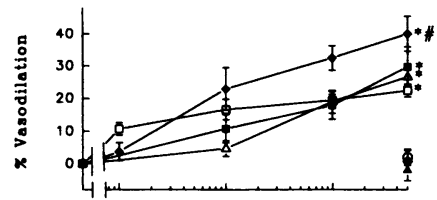

$\mathrm{B}$ nitroso-cysteine $(\bullet)$ on coronary blood flow, epicardial coronary vasodilation, and mean arterial pressure in anesthetized dogs. Control infusions included

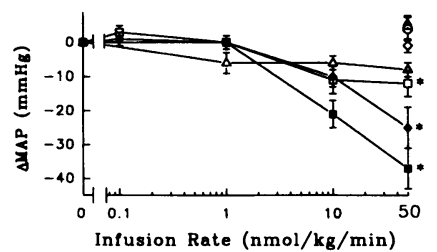

BSA $(\diamond), \mathrm{NaNO}_{2}(\Delta)$ and L-cysteine $(\mathrm{O})$. Intracoronary $S$-nitrosoBSA produced a dosedependent increase in coronary blood flow $(A)$

that was less pronounced $(P<0.05)$ than that of sodium nitroprusside, acetylcholine, and $S$-nitroso-cysteine. $S$-nitroso-BSA produced dose-dependent epicardial coronary vasodilation $(B)$ equivalent to acetylcholine and sodium nitroprusside but was less effective than $S$ nitroso-cysteine $(P<0.05)$. Intracoronary infusion of $S$-nitroso-BSA was not associated with a significant change in mean arterial pressure $(C)$ while acetylcholine, sodium nitroprusside, and $S$-nitroso-cysteine all produced a significant drop in mean arterial pressure $(P<0.05)$. ${ }^{*} P>0.05$ vs. control ${ }^{*} P<0.05$ vs. $S$-nitroso-BSA.

times, were compared with analysis of variance followed by a NeumanKeuls comparison. Statistical significance was accepted if the null hypothesis was rejected with a $P<0.05$.

\section{Results}

The coronary vascular response to $S$-nitroso-BSA. Changes in coronary blood flow, coronary artery diameter, and mean arterial pressure in response to intracoronary $S$-nitroso-BSA, acetylcholine, $S$-nitroso-cysteine, and nitroprusside are shown in Fig. 1. $S$-nitroso-BSA produced a dose-dependent increase in coronary blood flow of $165 \% \pm 24 \%$ at the maximal dose $(P$ $<0.05$ ), while acetylcholine, nitroprusside, and $S$-nitroso-cysteine exhibited increases of $483 \% \pm 55 \%(P<0.05), 315 \% \pm 82 \%$ $(P<0.05)$, and $475 \% \pm 66 \%(P<0.05)$, respectively (Fig. $1 A)$. The increases in coronary blood flow caused by $S$-nitroso-BSA and sodium nitroprusside were similar, while the responses produced by acetylcholine and $S$-nitroso-cysteine differed significantly from both $S$-nitroso-BSA $(P<0.05)$ and sodium nitroprusside $(P<0.05)$. No response was observed with the intracoronary infusion of BSA, $\mathrm{NaNO}_{2}$, or L-cysteine.

All four agents demonstrated dose-dependent coronary vasodilation in response to intracoronary administration (Fig. 1 $B$ ). $S$-nitroso-BSA, acetylcholine, and sodium nitroprusside all produced significant maximal coronary vasodilation of $27 \% \pm 3 \%(P<0.05), 20 \% \pm 2 \%(P<0.05)$, and $30 \% \pm 6 \%(P$ $<0.05)$, respectively, with no significant differences among the dose response curves of each agent. $S$-nitroso-cysteine produced significant coronary vasodilation of $40 \% \pm 7.8 \%$ ( $P$ $<0.05)$, which was significantly greater than any of the other agents $(P<0.05)$. BSA, L-cysteine, and $\mathrm{NaNO}_{2}$ had no effect on coronary vasodilation. A comparison of Fig. $1, A$ and $B$ reveals that $S$-nitroso-BSA produces epicardial coronary vasodilation comparable to that of acetylcholine and sodium nitro- 

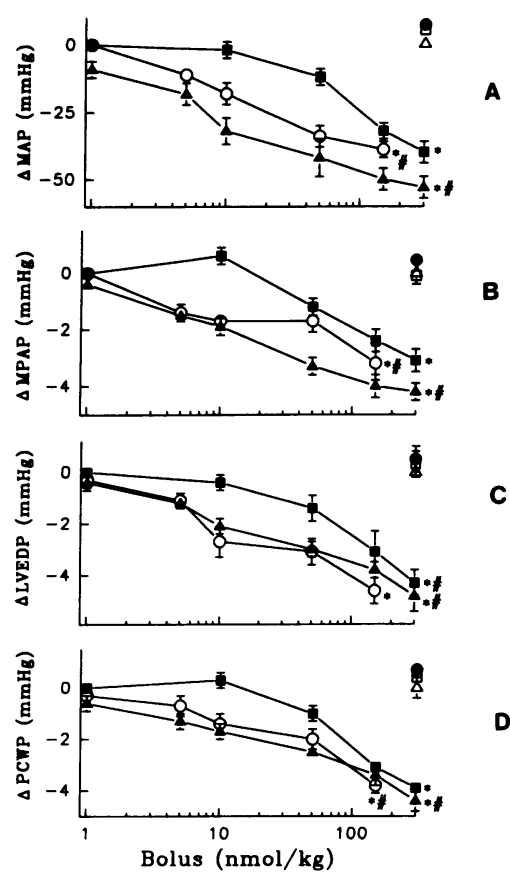

Figure 2. The systemic vascular response to $S$ nitroso-BSA. ( $A$ ) Mean arterial pressure, $(B)$ mean pulmonary artery pressure, $(C)$ left ventricular end-diastolic pressure, and $(D)$ mean pulmonary capillary wedge pressure in response to $S$-nitroso-BSA (匹), nitroglycerin (O), and $S$-nitroso-cysteine $(\triangle)$ given as an intravenous bolus. Controls

D included BSA ( $\square$ ), $\mathrm{NaNO}_{2}(\Delta)$, and L-cysteine $(\bullet) .{ }^{*} P<0.05$ vs. control, ${ }^{*} P<0.05$ vs. $S$-nitroso-BSA.

prusside while at the same time producing a smaller increase in estimated coronary blood flow.

The changes in mean arterial pressure in response to the intracoronary infusion of $S$-nitroso-BSA, sodium nitroprusside, $S$-nitroso-cysteine, and acetylcholine are shown in Fig. 1 $C$. Sodium nitroprusside produced the most significant reduction in mean arterial pressure $(37 \pm 6 \mathrm{mmHg} ; P<0.05)$, while acetylcholine, $S$-nitroso-cysteine, and $S$-nitroso-BSA produced less significant reductions $(11 \pm 4 \mathrm{mmHg}, P<0.05 ; 25 \pm 6$ $\mathrm{mmHg}, P<0.05$; and $6 \pm 2 \mathrm{mmHg}, P=\mathrm{NS}$, respectively).

Increases in coronary blood flow in response to $S$-nitrosoBSA were significantly more prolonged than those observed for any of the other agents $(P<0.05)$. Intracoronary $S$-nitrosoBSA increased coronary blood flow for a period of $587 \pm 93 \mathrm{~s}$, compared to $59 \pm 6,246 \pm 16$, and $19 \pm 2$ s for acetylcholine, $S$ nitroso-cysteine, and sodium nitroprusside, respectively. These values were recorded after the intracoronary infusion of each agent at the maximal dose.

$S$-nitroso-BSA and systemic hemodynamic parameters. The response of mean arterial pressure, mean pulmonary artery pressure, left ventricular end-diastolic pressure, and mean pulmonary capillary wedge pressure in response to the intravenous bolus administration of $S$-nitroso-BSA, nitroglycerin, and $S$-nitroso-cysteine are shown in Fig. 2. $S$-nitroso-BSA, nitroglycerin, and $S$-nitroso-cysteine all produced profound dosedependent decreases in mean arterial pressure of $40 \pm 5 \mathrm{mmHg}$ $(P<0.05), 37 \pm 2 \mathrm{mmHg}(P<0.05)$, and $53 \pm 4 \mathrm{mmHg}(P$ $<0.05$ ), respectively, at the maximal doses delivered (Fig. 2 $A$ ). On a molar basis, the response to $S$-nitroso-BSA was seven and a half-fold less potent than that of nitroglycerin and 10fold less potent than that of $S$-nitroso-cysteine $\left(\mathrm{EC}_{50}=75 \mathrm{vs}\right.$. 10 and $7.5 \mathrm{nmol} / \mathrm{kg}$, respectively $[P<0.05])$.

Intravenous bolus $S$-nitroso-BSA produced similar effects in the pulmonary circulation demonstrating dose-dependent reductions in mean pulmonary artery pressure (Fig. $2 \mathrm{~B}$ ). $S$ Nitroso-BSA was approximately ninefold less potent, on a molar basis, in reducing mean pulmonary artery pressure than nitroglycerin or $S$-nitroso-cysteine $\left(\mathrm{EC}_{50}=70\right.$ vs. 8 and 9 $\mathrm{nmol} / \mathrm{kg}$, respectively, $P<0.05)$.

The effects of intravenous bolus $S$-nitroso-BSA on left ventricular end-diastolic pressure and pulmonary capillary wedge pressure were similar (Fig. 2, $C$ and $D$ ). $S$-nitroso-BSA produced significant dose-dependent reductions in both left ventricular end diastolic pressure $(P<0.05)$ and mean pulmonary capillary wedge pressure $(P<0.05)$ with an $\mathrm{EC}_{50}$ of $\sim 70$ $\mathrm{nmol} / \mathrm{kg}$, which was sevenfold less potent than either nitroglycerin or $S$-nitroso-cysteine on a molar basis $(P<0.05)$.

The changes in mean arterial pressure seen with $S$-nitrosoBSA were more prolonged than those noted with nitroglycerin. Intravenous bolus $S$-nitroso-BSA (300 $\mathrm{nmol} / \mathrm{kg}$ ) reduced mean arterial pressure for a period of $15 \pm 3 \mathrm{~min}$, while equipotent doses of nitroglycerin $(150 \mathrm{nmol} / \mathrm{kg}$ ) and $S$-nitroso-cysteine $(100 \mathrm{nmol} / \mathrm{kg})$ reduced mean arterial pressure for only $3 \pm 1 \mathrm{~min}$ and $8 \pm 3 \mathrm{~min}$, respectively (both $P<0.05$ vs. $S$-nitroso-BSA). This prolonged duration of action of $S$-nitrosoBSA was observed throughout the dose range tested and was not explained by the degree of arterial pressure reduction. Representative tracings of mean arterial pressure in response to $S$-nitroso-BSA, $S$-nitroso-cysteine, and nitroglycerin are shown in Fig. 3.

The half-life of $S$-nitroso-cysteine in an aqueous oxygencontaining environment is reported to be $\sim 15 \mathrm{~s}(19)$. In this regard, the (relative) duration of action of $S$-nitroso-cysteine reported here ( $8 \pm 3 \mathrm{~min}$ ) appears inconsistent with these observations. We evaluated the potential role of NO transfer among plasma thiol species as a possible explanation for these discrepant observations. Before the administration of any $S$-nitrosocysteine, the plasma concentrations of protein and low molecular weight $S$-nitrosothiols were 0.71 and $0.022 \mu \mathrm{M}$, respectively. After the administration of $S$-nitroso-cysteine $\mathbf{0 . 5 2}$ $\mu \mathrm{M})$, these values increased twofold to 1.22 and $0.039 \mu \mathrm{M}$ suggesting the in vivo equilibration of $\mathrm{NO}$ among protein and low molecular weight thiol species. We were also able to demonstrate in vitro the transfer of NO from $S$-nitroso-albumin to L-cysteine (Fig. 4), supporting the proposed mechanism for our in vivo observations.

Both $S$-nitroso-BSA and nitroglycerin had significant effects on the indices of myocardial contractility and relaxation (Fig. 5). $S$-nitroso-BSA, $S$-nitroso-cysteine, and nitroglycerin produced dose-dependent decreases in maximal $\mathrm{dP} / \mathrm{dt}$ of

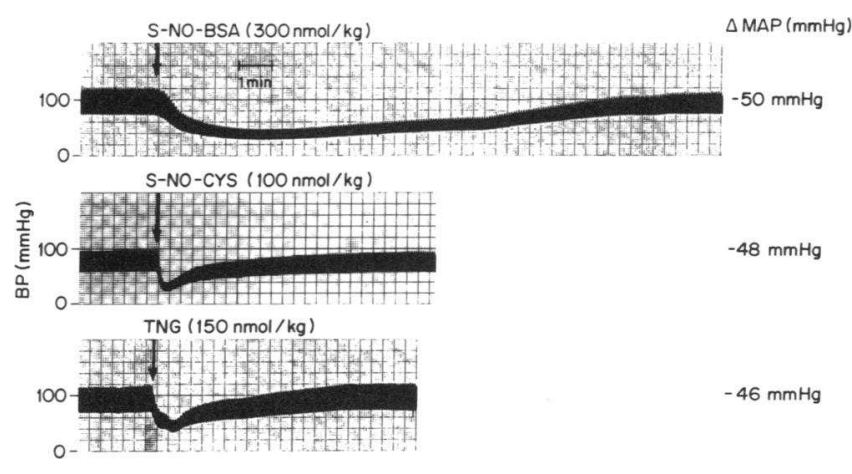

Figure 3. The time course of arterial pressure reduction in response to $S$-nitroso-BSA, nitroglycerin, and $S$-nitroso-cysteine. Representative tracings of mean arterial pressure after the intravenous bolus administration of nitroglycerin ( $T N G), S$-nitroso-cysteine ( $S N O-C Y S)$, and $S$-nitroso-BSA ( $S N O-B S A)$ at equipotent doses. 


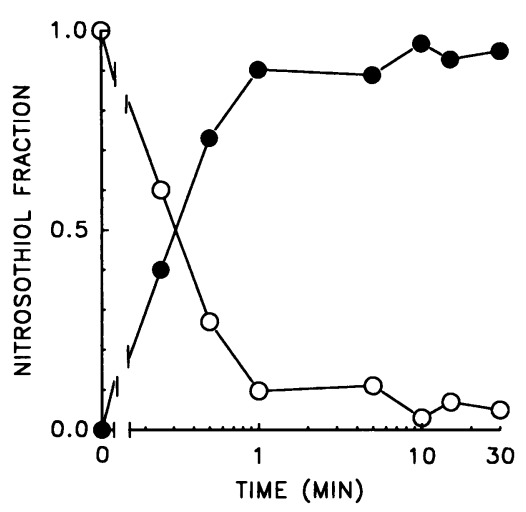

centration was determined by the difference $b$ teine concentration and total S-nitrosothiol concentration using the Saville reaction.

$581 \pm 101 \mathrm{mmHg} / \mathrm{sec}(P<0.05), 890 \pm 124 \mathrm{mmHg} / \mathrm{sec}(P$ $<0.05)$ and $464 \pm 63 \mathrm{mmHg} / \mathrm{sec}(P<0.05)$, respectively, at the maximal doses (Fig. $5 A$ ). Nitroglycerin, $S$-nitroso-cysteine, and $S$-nitroso-BSA also produced marked dose-dependent reductions in peak $-\mathrm{dP} / \mathrm{dt}$ of $1,008 \pm 156 \mathrm{mmHg} / \mathrm{sec}(P$ $<0.05), 1,740 \pm 159 \mathrm{mmHg} / \mathrm{sec}(P<0.05)$, and $859 \pm 134$ $\mathrm{mmHg} / \mathrm{sec}(P<0.05)$, respectively, again at the maximal doses tested (Fig. $5 \mathrm{~B}$ ). On a molar basis, $S$-nitroso-BSA proved $\sim 10$-fold less potent than nitroglycerin in reducing both $\mathrm{dP} / \mathrm{dt}$ and $-\mathrm{dP} / \mathrm{dt}\left(\mathrm{EC}_{50}=100\right.$ vs. $10 \mathrm{nmol} / \mathrm{kg}[P<0.05]$ and 90 vs. $9 \mathrm{nmol} / \mathrm{kg}[P<0.05]$, respectively). Furthermore, $S$-nitroso-BSA was $\sim 10$-fold less potent than $S$-nitroso-cysteine in reducing $\mathrm{dP} / \mathrm{dt}$, and 100 -fold less potent than $S$-nitroso-cysteine in reducing $-\mathrm{dP} / \mathrm{dt}\left(\mathrm{EC}_{50}=100 \mathrm{vs} .10 \mathrm{nmol} / \mathrm{kg}\right.$ $[P<0.05]$ and 90 vs. $0.9 \mathrm{nmol} / \mathrm{kg}[P<0.05]$, respectively).

The effects of $S$-nitroso-BSA and nitroglycerin given as 5-minute infusions on mean arterial pressure, mean pulmonary artery pressure, left ventricular end-diastolic pressure, and mean pulmonary capillary wedge pressure are shown in Fig. 6. $S$-nitroso-BSA and nitroglycerin again demonstrated profound dose-dependent effects on mean arterial pressure producing reductions of $40 \pm 5 \mathrm{mmHg}(P<0.05)$ and $37 \pm 2 \mathrm{mmHg}(P$ $<0.05$ ), respectively at the maximally tolerated doses (Fig. 6

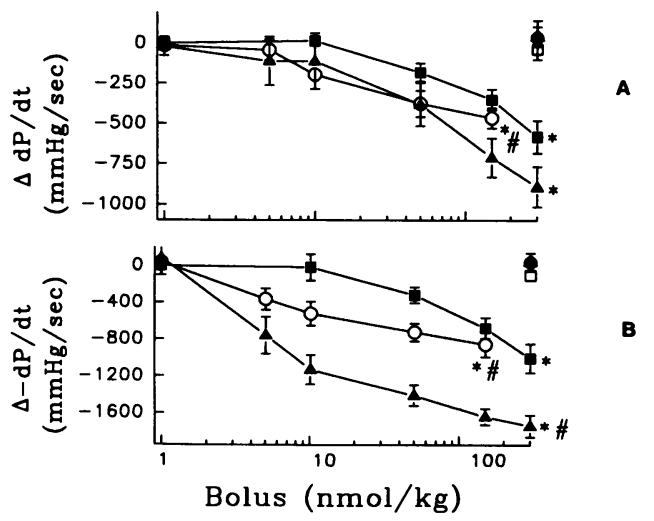

Figure 5. Myocardial contraction and relaxation in response to $S$-nitroso-BSA. Changes in indices of myocardial contractility $(A)$ and relaxation $(B)$ in response to $S$-nitroso-BSA $(\square)$, nitroglycerin $(O)$, and $S$-nitroso-cysteine $(\triangle)$ administered as a rapid intravenous bolus. Controls included BSA ( $\square), \mathrm{NaNO}_{2}(\bullet)$, and L-cysteine $(\Delta) .{ }^{*} P$ $<0.05$ vs. control, ${ }^{*} P<0.05$ vs. $S$-nitroso-BSA.

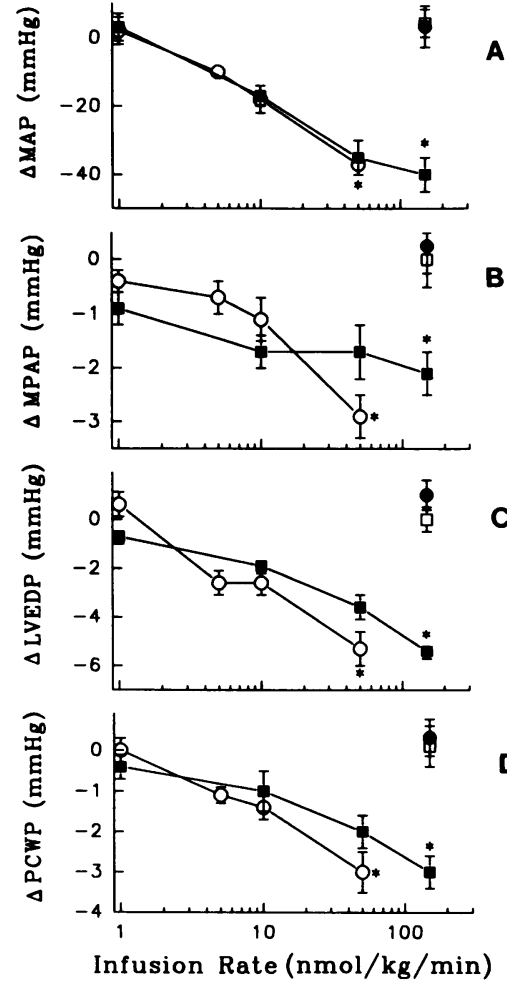

A

Figure 6. The systemic vascular response to $S$ nitroso-BSA administered as a five-minute infusion. $(A)$ Mean arterial pressure, $(B)$ mean pulmonary artery pressure, $(C)$ left ventricular end-diastolic pressure, and $(D)$ mean pulmonary capillary wedge pressure in response to $S$-nitroso-BSA (a) and nitroglycerin (o) given as a 5 -min infusion. Controls included BSA ( $\square$ ) and $\mathrm{NaNO}_{2}(\bullet) .{ }^{*} P<0.05$ vs. control.

A). In contrast to the responses seen with bolus administration, the dose-response curves for both agents administered by intravenous infusion were not significantly different with both agents exhibiting an $\mathrm{EC}_{50}$ of $\sim 10 \mathrm{nmol} / \mathrm{kg}$ per min. In a similar fashion, $S$-nitroso-BSA and nitroglycerin both demonstrated significant dose-dependent reductions in mean pulmonary artery pressure, left ventricular end-diastolic pressure, and mean pulmonary capillary wedge pressure with no significant differences in the dose-response curves between agents (Fig. 6, B-D).

Nitroglycerin and $S$-nitroso-BSA given as 5-min infusions demonstrated effects on left ventricular contractility and relaxation similar to those observed when the agents were given by bolus administration (Fig. 7). Nitroglycerin produced a dosedependent decrease in peak $\mathrm{dP} / \mathrm{dt}$ of $623 \pm 132 \mathrm{mmHg} / \mathrm{s}(P$ $<0.05)$ at the maximally tolerated dose, while $S$-nitroso-BSA produced a decrease of $767 \pm 135 \mathrm{mmHg} / \mathrm{sec}(P<0.05$; Fig. 7 $A)$. Nitroglycerin and $S$-nitroso-BSA produced more pronounced effects on peak $-\mathrm{dP} / \mathrm{dt}$ with dose-dependent decreases of $998 \pm 146 \mathrm{mmHg} / \mathrm{sec}(P<0.05)$ and $1,119 \pm 162 \mathrm{mmHg} / \mathrm{sec}$ $(P<0.05)$, respectively, at the maximally tolerated doses (Fig. $7 \mathrm{~B}$ ). Again, in contrast to the effects with bolus administration, the dose-response curves of nitroglycerin and $S$-nitrosoBSA given as 5-min infusions with respect to $\mathrm{dP} / \mathrm{dt}$ and $-\mathrm{dP} / \mathrm{dt}$ did not differ significantly.

S-nitroso-BSA and platelet function. S-nitroso-BSA demonstrated profound dose-dependent platelet inhibition as measured by both in vivo and in vitro techniques. The effect of $S$-nitroso-BSA on template bleeding time is shown in Fig. 8. The administration of $S$-nitroso-BSA as a 5-min infusion produced a dose-dependent, fivefold increase in the template bleeding time from $1.9 \pm 0.13$ to $10.93 \pm 0.6 \mathrm{~min}(P<0.01)$. This increase in bleeding time was associated with a dose-de- 


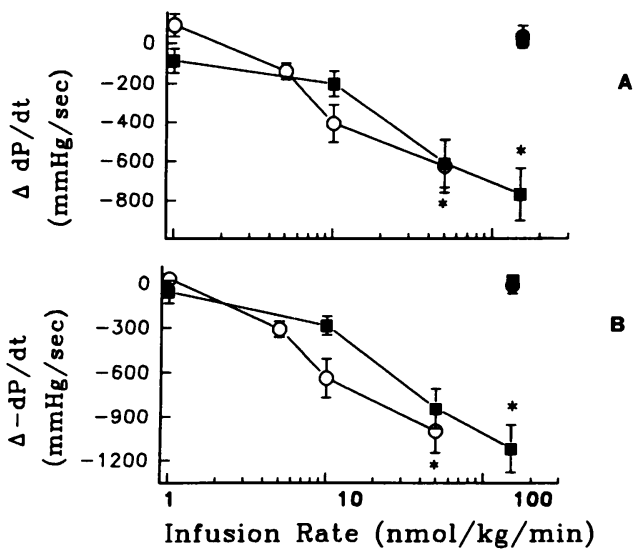

Figure 7. Myocardial contraction and relaxation in response to $S$-nitroso-BSA infusion. Changes in indices of myocardial contractility (A) and relaxation (B) in response to $S$-nitroso-BSA ( $\bullet$ ), nitroglycerin $(O)$, administered as a 5-min intravenous infusion. Controls included BSA ( $\square$ ) and $\mathrm{NaNO}_{2}(\bullet) .{ }^{*} P<0.05$ vs. control.

pendent inhibition of ex vivo ADP-induced platelet aggregation (Fig. 8). Similar results were obtained with collagen-induced platelet aggregation (data not shown). BSA ( $300 \mathrm{nmol} /$ $\mathrm{kg}$ per min ) as well as $\mathrm{NaNO}_{2}(300 \mathrm{nmol} / \mathrm{kg}$ per min $)$ did not demonstrate any effect on bleeding time (Fig. $8 \mathrm{~A}$ ).

\section{Discussion}

We have recently reported the observation that oxides of nitrogen can combine with protein-bound thiol groups to form stable $S$-nitrosothiols (22). Furthermore, we have shown that this reaction occurs under physiologic conditions producing stable $S$-nitroso-proteins that are biologically active, exhibiting vasodilatory and antiplatelet properties in vitro through a cyclic-GMP dependent mechanism (22). The data presented here extend these observations to an in vivo model and demonstrate that BSA can combine with $\mathrm{NO}$ (probably as $\mathrm{NO}^{+}$, the nitrosonium ion) to form $S$-nitroso-BSA that posseses EDRFlike properties including $(a)$ significant epicardial coronary artery vasodilation; $(b)$ a less prominent but significant increase in coronary blood flow; $(c)$ systemic venous and arterial vasodilation; and $(d)$ in vivo dose-dependent platelet inhibition. Furthermore, the long chemical half-life of $S$-nitroso-BSA is reflected in its relative duration of action compared with more typical nitrovasodilators, such as sodium nitroprusside, nitroglycerin, and $S$-nitroso-cysteine.

Our data demonstrate that $S$-nitroso-BSA induces epicardial coronary vasodilation comparable to that of sodium nitroprusside, acetylcholine, and $S$-nitroso-cysteine, but is less effective as a vasodilator of coronary resistance vessels than these agents. Nitroglycerin acts predominantly upon large diameter coronary vessels with little effect on resistance vessels, while nitroprusside exerts its effect preferentially on smaller diameter coronary vessels (33). In this regard, $S$-nitroso-BSA appears to have an effect that is somewhat similar to that of nitroglycerin in the coronary circulation. The mechanism(s) underlying this phenomenon is unclear but may be related to the method by which BSA-bound nitric oxide is delivered from plasma to intracellular effector sites. If, for example, the denitrification of
$S$-nitroso-BSA requires the presence of a specific cell surface molecular "acceptor," then the differential effects of $S$-nitrosoBSA on small and large coronary vessels could be explained by the preferential distribution of these cell surface species on the conductance vessel endothelium. Evidence for the interaction of NO with membrane surface groups in the context of a biologic response has been demonstrated (34). Other possibilities for consideration might include the differential distribution of intracellular "transport" species required for the delivery of BSA-bound nitric oxide to guanylyl cyclase, or simply the differential kinetics of relaxation between small and large coronary arteries.

The data presented here also show that $S$-nitroso-BSA, when given as an intravenous bolus, is $\sim 7$ - to 10 -fold less potent on a molar basis than both $S$-nitroso-cysteine and nitroglycerin. However, when administered as a 5-min infusion, $S$-nitroso-BSA and nitroglycerin appear to be equipotent. One possible explanation for this observation may be the difference in relaxation kinetics between nitroglycerin and $S$-nitrosoBSA. In organ chamber experiments using isolated rabbit aortic rings precontracted with $1 \mu \mathrm{M}$ norepinephrine, nitroglycerin exhibits rapid, transient, dose-dependent relaxations while $S$-nitroso-BSA produces relaxations that are slower in onset and more prolonged (22). It is conceivable that when given as a bolus, the rate of NO release from $S$-nitroso-BSA may be relatively slow compared to the rates of both NO inactivation and $S$-nitroso-BSA metabolism, thereby limiting the degree of guanylate cyclase activation by NO. When given as a 5 -min infusion, however, the constant supply of slowly released $S$-nitroso-BSA may afford a greater degree of sustained guanylyl cyclase activation, and, therefore, provide a more potent effect.

Nitric oxide is an highly reactive species formed in a cellular environment replete with coreactants and enzymes capable of stabilizing its activity or promoting its degradation $(17,19$, 20 ). In this regard, the chemical form in which NO is transported to target sites in vivo remains unclear. Biologic thiols react readily with oxides of nitrogen forming $S$-nitrosothiols, which preserve the bioactivity of NO and prolong its half-life $(9,19,35,36)$. The biologic importance of these findings is

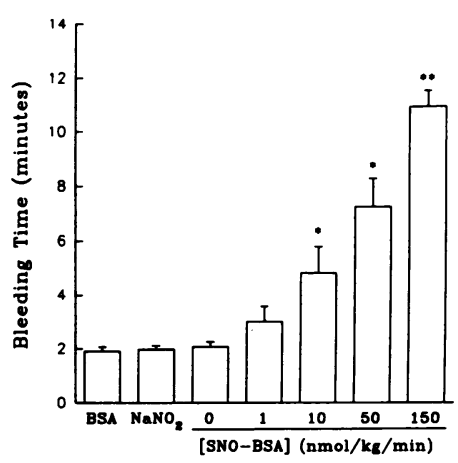

A

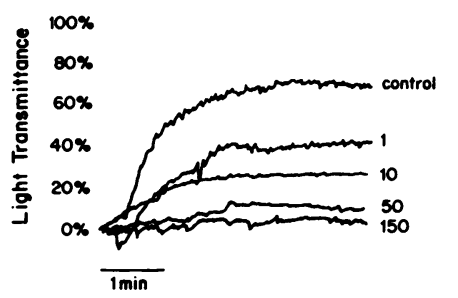

Figure 8. The platelet inhibitory effects of $S$ nitroso-BSA. Bleeding time $(A)$ and ex vivo ADP-induced platelet aggregation $(B)$ in response to $S$-nitroso-BSA

$B$ given as a 5-min infusion. Bleeding times and platelet aggregations were performed fifteen minutes after the end of each infusion. ${ }^{*} P$ $<0.05$ and $* * P<0.01$ vs. BSA, $\mathrm{NaNO}_{2}$, and control dose. 
exemplified by the observations that low molecular weight $S$ nitrosothiols are more stable than NO (19), possess EDRF-like vasorelaxant properties (20), and inhibit platelet aggregation via a cyclic GMP-dependent mechanism (37). Furthermore, we have shown that low molecular weight thiols prolong the half life of EDRF and, thereby, potentiate the antiplatelet and vasorelaxant properties of EDRF through a cyclic GMP-dependent mechanism $(33,37,38)$. Our recent demonstration of $S$-nitrosothiol formation from endogenously derived NO with proteins as a thiol source provides important evidence for the role of $S$-nitrosothiols in the action and metabolism of EDRF (22). Moreover, the results reported here provide further evidence that protein thiol can serve to form an NO adduct, preserving both its bioactivity and increasing its half-life in vivo. It should be noted, however, that the prolonged (minutes) bioactivity of protein bound NO in vivo conflicts with currently held notions of NO reactivity, biologic activity, and regulatory function. This discrepancy, however, may be mitigated by our demonstration of nitric oxide transfer among available thiol species.

The data presented here support the hypothesis that protein-bound thiol can participate in the action of EDRF, although the mechanism by which NO is delivered from serum albumin in the plasma milieu to intracellular target sites remains unclear. Serum albumin, although abundant in plasma, does not readily traverse endothelial cell membranes. Furthermore, the relatively long half life of $S$-nitroso-BSA ( $12 \mathrm{~h}$ at $37^{\circ} \mathrm{C}, \mathrm{pH} 7.4$, aqueous solution, $95 \% \mathrm{O}_{2}[22]$ ) argues against the spontaneous release of $\mathrm{NO}$ as the mechanism of smooth muscle relaxation, suggesting that facilitated release or transfer of NO occurs. In this regard, the findings of Kowaluk and Fung (36) are of particular interest. These investigators observed that the vascular relaxant potencies of $S$-nitrosothiols were not correlated with either the spontaneous release of nitric oxide or their relative lipophilicity. It seems likely, therefore, that the vasorelaxant properties of $S$-nitrosothiols are modulated through some other mechanism, such as the transfer of NO to a "carrier" molecule capable of traversing the cell membrane or, alternatively, direct interaction with reactive membranebound species such as sulfhydryl groups, iron-sulfur containing proteins, or surface protein-bound thiols (34).

There is ample evidence to support the possible involvement of NO exchange reactions in the action and metabolism of $S$-nitroso species. Oae and colleagues (39) demonstrated the exchange of nitric oxide among reactive sulfhydryl species under physiologic conditions, while Chong and Fung (40) described the catalysis of $N$-acetylcysteine-mediated denitrification of nitroglycerin by serum albumin. Furthermore, Keen and co-workers (41) demonstrated the enzymatic denitrification of nitroglycerin by glutathione-S-transferase, and Bennett and colleagues provided evidence for the catalysis of nitroglycerin denitrification by hemoproteins (42). Finally, our data demonstrating the equilibration of NO among the available thiol species in plasma and in vitro also support the involvement of NO/thiol exchange reactions in the action of $S$-nitroso species.

In summary, the results presented here demonstrate that the free sulfhydryl group of serum albumin, located at cysteine 34 , is capable of combining with nitric oxide to form a uniquely stable $S$-nitrosothiol that possesses both vasorelaxant and platelet inhibitory properties in vivo reminiscent of authentic EDRF. These results support the hypothesis that protein- bound sulfhydryl species can participate in the action and metabolism of EDRF by prolonging its half-life and preserving its biologic activity.

\section{Acknowledgments}

This work was supported in part by National Institutes of Health grants HL40411 and HL43344, a Grant-in-Aid from the American Heart Association with funds contributed in part from the Massachusetts Affiliate, and by a Merit Review Award from the Veterans Administration. Daniel Simon is the Samuel A. Levine Fellow of the Massachusetts Affiliate of the American Heart Association, Jonathan Stamler is the recipient of a Clinician Investigator Award from the National Institutes of Health (K08HL02582), Joseph Vita is the recipient of a Clinician Scientist Award (91004100) from the American Heart Association, and Joseph Loscalzo is the recipient of a Research Career Development Award from the National Institutes of Health (K04HL02273).

\section{References}

1. Furchgott, R. F., and J. V. Zawadzki. 1980. The obligatory role of endothelial cells in the relaxation of arterial smooth muscle by acetylcholine. Nature (Lond.). 288:373-376.

2. Furchgott, R. F. 1983. Role of endothelium in responses of vascular smooth muscle. Circ. Res. 35:557-573.

3. Radomski, R. A., R. M. J. Palmer, and S. Moncada. 1987. Comparative physiology of endothelium-derived relaxing factor, nitric oxide, and prostacyclin in platelets. Br. J. Pharmacol. 92:181-187.

4. Palmer, R. M. J., A. G. Ferrige, and S. Moncada. 1987. Nitric oxide release accounts for the biologic activity of endothelium-derived relaxing factor. Nature (Lond.). 327:524-526.

5. Lancaster, J. R., Jr., and J. B. Hibbs, Jr. 1990. EPR demonstration of iron-nitrosyl complex formation by cytotoxic activated macrophages. Proc. Natl. Acad. Sci. USA. 87:1223-1227.

6. Bunton, C. A., D. R. Llewellyn, and G. Stedman. 1957. In Recent Aspects of the Inorganic Chemistry of Nitrogen. Special Publication No. 10, The Chemical Society, London). 113-120.

7. Saran, M., C. Michel, and W. Bors. 1990 . Reaction of NO with $\mathrm{O}_{2}$. Implications for the reaction of endothelium-derived relaxing factor (EDRF). Free Radical Res. Commun. 10:221-226.

8. Stamler, J., and J. Loscalzo. 1990. Nitrosylation of homocysteine as a novel antithrombotic mechanism. Clin. Res. 38:246a.

9. Ignarro, L. J., J. C. Edwards, D. Y. Greutter, B. K. Barry, and C. A. Greutter. 1980. Possible involvement of S-nitrosothiols in the activation of guanylate cyclase by nitroso compounds. FEBS (Fed. Eur. Biochem. Soc.) Lett. 11:275278.

10. Loscalzo, J. 1985. $N$-acetylcysteine potentiates inhibition of platelet aggregation by nitroglycerin. J. Clin. Invest. 76:703-708.

11. Downes, M. J., M. W. Edwards, T. S. Elsey, and C. L. Walters. 1976. Determination of a non-volatile nitrosamine by using denitrosylation and a chemiluminescence analyzer. Analyst. 101:742-748.

12. Pryor, W. A., and J. W. Lightsey. 1981. Mechanisms of nitrogen dioxide reactions: initiation of lipid peroxidation and the production of nitrous acid. Science (Wash. DC.) 214:435-437.

13. Palmer, R. M. J., D. S. Ashton, and S. Moncada. 1988. Vascular endothelial cells synthesize nitric oxide from L-arginine. Nature (Lond.). 333:664-666.

14. Buga, G. M., M. E. Gold, K. S. Woods, G. Choudhuri, and L. J. Ignarro. 1989. Endothelium-derived nitric oxide relaxes nonvascular smooth muscle. Eur. J. Pharmacol. 161:61-72.

15. Hoeffner, U., C. Boulanger, and P. M. Vanhoutte. 1989. Proximal and distal dog arteries respond differently to basal EDRF but not NO. Am. J. Physiol. 256:H828-H831.

16. Kelm, M., and J. Schrader. 1990. Control of coronary vascular tone by nitric oxide. Circ. Res. 66:1561-1575.

17. Ignarro, L. J. 1989. Biological actions and properties of endothelium-derived nitric oxide formed and released from artery and vein. Circ. Res. 65:1-21.

18. Cox, D. A., J. A. Vita, C. B. Treasure, R. D. Fish, R. W. Alexander, P. Ganz, and A. P. Selwyn, A. P. 1989. Atherosclerosis impairs flow-mediated dilation of coronary arteries in humans. Circulation. 80:458-465.

19. Ignarro, L. J., H. Lipton, J. C. Edwards, W. H. Barricos, A. L. Hyman, P. J. Kadowitz, and C. A. Greutter. 1981. Mechanism of vascular smooth muscle relaxation by organic nitrates, sodium nitroprusside, and nitric oxide: evidence for the involvement of S-nitrosothiols as active intermediates. J. Pharmacol. Exp. Ther. 218:739-749.

20. Myers, P. R., R. L. Minor, Jr., R. Guerra, Jr., J. N. Bates, and D. G. Harrison. 1990. Vasorelaxant properties of the endothelium-derived relaxing fac- 
tor more closely resemble S-nitrosocysteine than nitric oxide. Nature (Lond.). 345:161-163.

21. Jocelyn, P. C. 1972. Thiols and disulfides in blood. In Biochemistry of the SH Group. Academic Press Limited, London. 240-260.

22. Stamler, J. S., D. I. Simon, J. A. Osborne, M. E. Mullins, O. Jaraki, D. J. Singel, and J. Loscalzo. 1992. S-nitrosylation of proteins by nitric oxide: synthesis and characterization of novel biologically active compounds. Proc. Natl. Acad. Sci. USA. 89:444-448.

23. Saville, B. 1958. A scheme for the colorimetric determination of microgram amounts of thiols. Analyst. 83:670-672.

24. Bonnett, R., R. Holleyhead, B. L. Johnson, and E. W. Randall. 1975. Reaction of acidified nitrite solutions with peptide derivatives: evidence for nitrosamine and thionitrite formation from ${ }^{15}$ N N.M.R. studies. J. Chem. Soc. Perkin Trans. I. 2261-2264.

25. Byler, D. M., D. K. Gosser, and H. Susi. 1983. Spectroscopic estimation of the extent of S-nitrosothiol formation by nitrite action on sulfhydryl groups. $J$. Agric. Food Chem. 31:523-527.

26. Stamler, J. S., O. A. Jaraki, J. A. Osborne, D. I. Simon, J. F. Keaney, J. A. Vita, D. J. Singel, C. R. Valeri, and J. Loscalzo. 1992. Nitric oxide circulates in mammalian plasma primarily as an S-nitroso adduct of serum albumin. Proc. Natl. Acad. Sci. USA. 89:7674-7677.

27. Sandor, T., and J. R. Spears. 1985. Statistical considerations on the precision of assessing blood vessel diameter in cine coronary angiography. Comput. Biomed. Res. 18:531-543.

28. Ludmer, P. L., A. P. Selwyn, T. L. Shook, R. R. Wayne, G. H. Mudge, R. W. Alexander, and P. Ganz. 1986. Paradoxical acetylcholine induced coronary artery constriction in patients with coronary artery disease. $N$. Engl. J. Med. 315:1046-1051.

29. Nabel, E. G., P. Ganz, J. B. Gordon, R. W. Alexander, and A. P. Selwyn. 1988. Dilation of normal and constriction of atherosclerotic coronary arteries caused by cold pressor test. Circulation. 77:43-52.

30. Gordon, J. B., P. Ganz, E. G. Nabel, J. Zabede, G. H. Mudge, R. W. Alexander, and A. P. Selwyn. 1989. Atherosclerosis and endothelial function influence the coronary response to exercise. J. Clin. Invest. 83:1946-1952.

31. Vita, J. A., C. B. Treasure, E. G. Nabel, J. M. McLenachan, R. D. Fish A. C. Yeung, V. I. Vekshtein, A. P. Selwyn, and P. Ganz. 1990. The coronary vasomotor response to acetylcholine relates to risk factors for coronary artery disease. Circulation. 81:491-497.

32. Sibley, D. H., H. D. Millar, C. H. Hartley, and P. L. Whitlow. 1986. Subselective measurements of coronary blood flow velocity using a steerable Doppler catheter. J. Am. Coll. Cardiol. 8:1332-1340.

33. Macho, P., and S. F. Vatner. 1981. Effects of nitroglycerin and nitroprusside on large and small coronary vessels in conscious dogs. Circulation. 64:11011107.

34. Mordvintsev, P. I., V. G. Rudneva, A. F. Vanin, L. L. Shimkevitch, and B. I. Khorodov. 1986. Inhibition of platelet aggregation by dinitrosyl iron complexes with low molecular weight ligands. Biokhimyia. 51:1851-1857.

35. Cooke, J. P., J. S. Stamler, N. Andon, P. F. Davies, and J. Loscalzo. 1990 Flow-mediated endothelium dependent vasodilation is potentiated by $\mathrm{N}$-acetylcysteine. Am. J. Physiol. 28:H804-H812.

36. Kowaluk, E. A., and H.-L. Fung. 1990. Spontaneous liberation of nitric oxide cannot account for in vitro vascular relaxation by S-nitrosothiols. $J$. Pharm. Exp. Ther. 256:1256-1264.

37. Mellion, B. T., L. J. Ignarro, C. B. Meyers, E. H. Ohlstein, B. A. Ballot, A. L. Hyman, and P. J. Kadowitz. 1983. Inhibition of human platelet aggregation by S-nitrosothiols. Heme dependent activation of soluble guanylate cyclase and stimulation of cyclic GMP accumulation. Mol. Pharmacol. 23:653-654.

38. Stamler, J., M. E. Mendelsohn, P. Amarante, D. Smick, N. Andon, P. F Davies, J. P. Cooke, and J. Loscalzo. 1989. N-acetylcysteine potentiates platele inhibition by endothelium-derived relaxing factor. Circ. Res. 65:789-795.

39. Oae, S., Y. H. Kim, D. Fukushima, and K. Shinhama. 1978. New synthesis of thionitrites and their chemical reactivities. J. Chem. Soc. Perkin Trans. I. 913-917.

40. Chong, S., and H.-L. Fung. 1990. Thiol mediated catalysis of nitroglycerin degradation by serum proteins. Increase in metabolism was not accompanied by S-nitrosothiol production. Drug. Metab. Dispos. 18:61-67.

41. Keen, J. H., W. H. Habig, and W. B. Jakoby. 1976. Mechanism of the several activities of the glutathione S-transferases. J. Biol Chem 251:6183-6188.

42. Bennett, B. M., S. M. Kobus, J. F. Brien, K. Nakatsu, and G. S. Marks. 1987. Requirement for reduced, unliganded hemoprotein for the hemoglobinand myoglobin-mediated biotransformation of glyceryl trinitrate. J. Pharm. Exp. Ther. 237:629-635. 